\title{
Análisis de la curvatura radicular para definir tratamiento endodontico de molares inferiores. Universidad Nacional de Chimborazo, 2018
}

\section{Analysis of radicular curvature to define endodontic treatment of lower molars. Universidad Nacional de Chimborazo, 2018}

\section{Análise da curvatura radicular para definir tratamento endodôntico de molares inferiores. Universidade Nacional de Chimborazo, 2018}

Roddy M. Chávez-Sandoval ${ }^{\mathrm{I}}$

Verónica A. Guamán-Hernández II

vguaman@unach.edu.ec

Sandra M. Quisiguiña-Guevara ${ }^{\text {III }}$

smquisiguina@unach.edu.ec

Natalia A. Gavilanes-Bayas IV

natalia.gavilanes@unach.edu.ec

Cristian R. Sigcho-Romero V

roanle-33@hotmail.com

Recibido: 27 de noviembre de 2018 * Corregido: 18 de diciembre de $2018 *$ Aceptado: 20 de diciembre de 2018

I. Odontólogo.

II. Especialista en Endodoncia; Odontóloga; Docente Facultad de Ciencias de la salud, Carrera de Odontología, Universidad Nacional de Chimborazo.

III. Especialista en Estética y operatoria dental; Odontóloga; Docente Facultad de Ciencias de la salud, Carrera de Odontología, Universidad Nacional de Chimborazo.

IV. Especialista en Rehabilitación Oral, Odontóloga Docente Facultad de Ciencias de la salud, Carrera de Odontología, Universidad Nacional de Chimborazo.

V. Especialista en Prótesis fija, removible e implantoasistida dentobucomaxilar. Docente Facultad de Ciencias de la salud, Carrera de Odontología, Universidad Nacional de Chimborazo. 


\title{
Resumen
}

La curvatura radicular del primer y segundo molar inferior analizado mediante 50 radiografías periapicales con el método de Schneider en las provincias de Chimborazo, Tungurahua, Cotopaxi año 2018. Obtuvo como resultado mediante la utilización del programa AUTOCAD 2015 que la dirección en la vista mesial y distal fue recta en un $78 \%$ y $76 \%$. Concluyendo que la curvatura radicular determina el fracaso endodóntico por la clasificación severa en $66 \%$ y $74 \%$ en su raíz distal, mesial.

Palabras claves: Molares; Curvatura Radicular; Fracaso Endodóntico.

\begin{abstract}
The radicular curvature of the first and second lower molar analyzed by 50 peri-apical radiographs with the Schneider method in the provinces of Chimborazo, Tungurahua, Cotopaxi, 2018. It obtained as a result by using the AUTOCAD 2015 program that the direction in the mesial view and distal was $78 \%$ and $76 \%$ straight. Concluding that the root curvature determines the endodontic failure by the severe classification in $66 \%$ and $74 \%$ in its distal, mesial root.
\end{abstract}

Key words: Molars; Root Curvature; Endodontic Failure.

\section{Resumo}

A curvatura radicular do primeiro e segundo molar inferior analisada por 50 radiografias peri-apicais com o método de Schneider nas províncias de Chimborazo, Tungurahua, Cotopaxi, 2018. Obteve como resultado, utilizando o programa AUTOCAD 2015, que a direção no mesial vista e distal foi de $78 \%$ e $76 \%$ em linha reta. Concluindo que a curvatura da raiz determina a falha endodôntica pela classificação severa em $66 \%$ e $74 \%$ em sua raiz mesial distal.

Key words: Molares; Curvatura Raiz; Falha Endodôntica. 


\section{Introducción.}

La endodoncia es una especialidad odontológica que estudia la morfología, fisiología, patología de la pulpa dentaria y de los tejidos adyacentes; así como su correcto tratamiento para la conservación de piezas dentales; (1) El tratamiento endodóntico es necesario para la remoción de tejido pulpar infectado, realizando el protocolo para evitar reinfección.

Se debe considerar que los factores para un tratamiento endodontico exitoso son el estado de salud óptimo, reacción de cicatrización y anatomía de los conductos que pueden llegar a presentar curvas muy marcadas; La investigación se realiza en la Universidad Nacional de Chimborazo con la donación de 50 radiografías de la cátedra de Imagenología dictada por la Dra. Verónica Guamán.

Este trabajo de investigación determina el grado de la curvatura que presentan los primeros y segundos molares inferiores mediante el método de Schneider, este enuncia que un conducto curvo se lo debe dividir en dos segmentos, es decir con una línea que sigue eje longitudinal de la raíz desde el piso de la cámara pulpar y otra línea que va desde el ápice y el tercio apical de la raíz hacia oclusal, (2)clasificándose según el grado de las curvaturas radiculares en leve, moderado y severo.

El procedimiento para obtener la clasificación de la curvatura radicular se realiza en un gráfico en papel milimetrado y se obtiene el ángulo parcial de la curvatura de cada pieza dental, a continuación se determina el grado de las curvaturas usando el programa AutoCAD 2015 e incorporando las imágenes radiográficas para establecer su clasificación según el ángulo y el tipo de curvatura de cada raíz. (3)

La radiografía peri-apical es fundamental antes, durante y después del tratamiento; ya que lleva un orden, en el cual los detalles anatómicos, la longitud del conducto, la calidad de obturación,

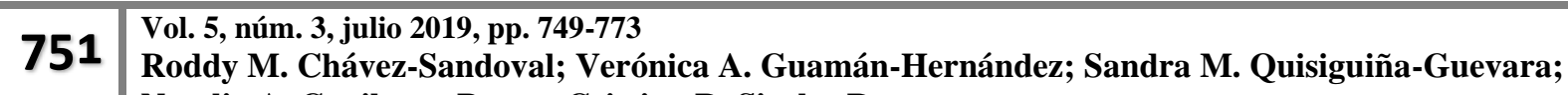
Natalia A. Gavilanes-Bayas; Cristian R. Sigcho-Romero
} 
la patología ósea y dental se puede identificar; no son método diagnóstico para una patología pulpar, sino es prueba complementaria para diagnosticar la patología peri-apical. (4)

La radiografía peri-apical facilita la evaluación dentaria de la región peri-apical en dos dimensiones: alto y ancho. Cuando se presenta la necesidad de evaluar una patología en la que debemos considerar la profundidad, es necesario realizar variaciones a las técnicas radiográficas tradicionales conocidas como técnicas de localización radiográficas. (4)

\section{Metodología.}

Tipo de investigación

Estudio investigativo de tipo transversal y observacional para conocer el grado, clasificación, tipo y curvatura según el tercio radicular con el método de Schneider.

\section{Población}

50 radiografías peri-apicales donadas de las provincias de Chimborazo, Tungurahua,

\section{Cotopaxi.}

Criterios de inclusión

a. Radiografías de primeros y segundos molares inferiores.

b. Radiografías sin errores radiográficos.

c. Radiografías de molares extraídos.

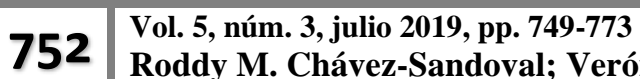




\section{Criterios de exclusión}

a. Radiografías de piezas que no sean las enfocadas a la investigación.

b. Radiografías con errores radiográficos.

c. Radiografías que no sean del año del estudio.

d. Radiografías deterioradas.

Técnicas e instrumentos

La técnica que se utilizó en el estudio fue observacional y como instrumento la ficha de registro de las 50 radiografías peri-apicales y para la interpretación de cuadros estadísticos en el programa estadístico IBM SPSS Versión 22.

\section{Resultados.}

Tabla 1.- Media, moda, desviación estándar

\begin{tabular}{|c|c|c|c|c|c|c|c|c|}
\hline & $\begin{array}{l}\text { Clasificación } \\
\text { Raíz } \\
\text { Distal } \\
\text { Schneider }\end{array}$ & $\begin{array}{l}\text { Clasificación } \\
\text { Raíz } \\
\text { Mesial } \\
\text { Schneider }\end{array}$ & $\begin{array}{l}\text { Tipo } \\
\text { curva } \\
\text { Vista } \\
\text { Distal }\end{array}$ & $\begin{array}{l}\text { Tipo } \\
\text { curva } \\
\text { Vista } \\
\text { Mesia } \\
1\end{array}$ & $\begin{array}{l}\text { Tipo curva } \\
\text { Vista } \\
\text { Vestibular } \\
\text { Raíz } \\
\text { Distal }\end{array}$ & $\begin{array}{l}\text { Tipo curva } \\
\text { Vista } \\
\text { Vestibular } \\
\text { Raíz } \\
\text { Mesial }\end{array}$ & $\begin{array}{l}\text { Tercio } \\
\text { Raíz } \\
\text { Distal }\end{array}$ & $\begin{array}{l}\text { Tercio } \\
\text { Raíz } \\
\text { Mesia } \\
1\end{array}$ \\
\hline \begin{tabular}{l|l|}
$\mathrm{N}$ & Válido \\
\end{tabular} & 50 & 50 & 50 & 50 & 50 & 50 & 50 & 50 \\
\hline Perdido & 0 & 0 & 0 & 0 & 0 & 0 & 0 & 0 \\
\hline Media & 2,64 & 2,74 & 1,38 & 1,28 & 1,36 & 1,90 & 2,36 & 1,86 \\
\hline Moda & 3 & 3 & 1 & 1 & 1 & 2 & 2 & 2 \\
\hline
\end{tabular}


Dom. Cien., ISSN: 2477-8818

Vol. 5, núm.3. julio., 2019, pp. 749-773

Análisis de la curvatura radicular para definir tratamiento endodontico de molares inferiores. Universidad Nacional de Chimborazo, 2018

\begin{tabular}{|l|l|l|l|l|l|l|l|l|}
\hline $\begin{array}{l}\text { Desviació } \\
\text { n estándar }\end{array}$ &, 525 &, 443 &, 725 &, 573 &, 485 &, 303 &, 631 &, 700 \\
\hline
\end{tabular}

Elaborado por: Roddy Chavez

Fuente: IBM SPSS versión 22.

\section{Ilustración 1.- Media}

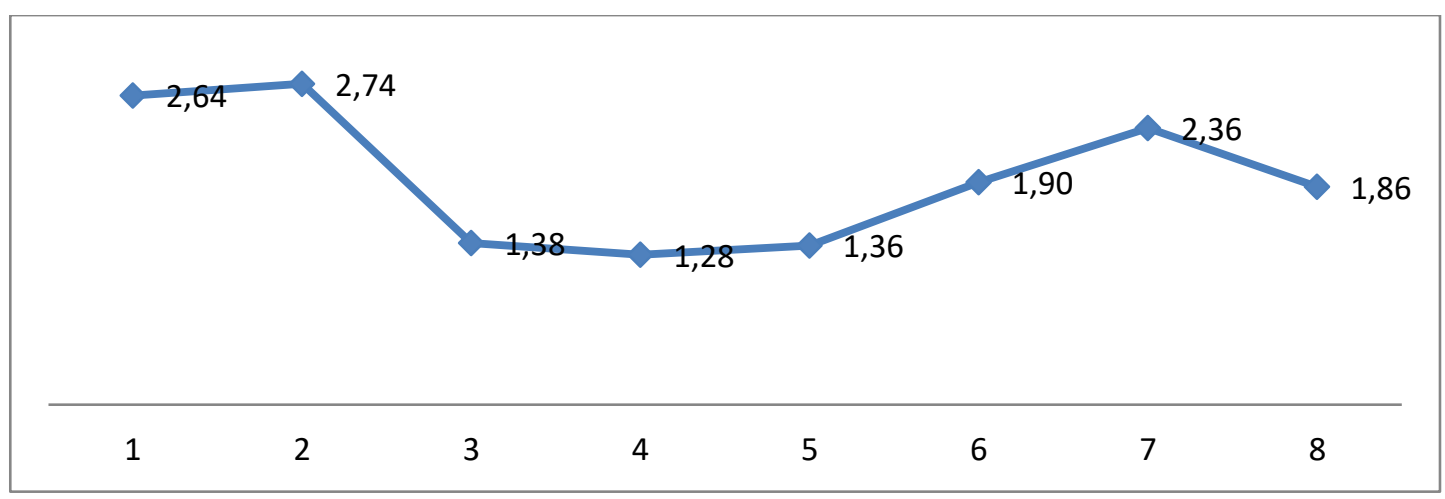

Elaborado por: Roddy Chavez

Fuente: IBM SPSS versión 22.

Descripción: La media en referencia a cada uno de los objetivos analizados corresponde a: clasificación de Schneider en la raíz distal con un 2.64 y raíz mesial 2.74, Tipo de curvatura vista distal, mesial es de raíz distal 1.38 y mesial 1.28, Tipo de curvatura vista vestibular raíz distal 1.36, Tipo de curvatura vista vestibular raíz mesial 1.90, Curvatura raíz distal según su tercio 2.36 y raíz mesial de 1.86 .

Análisis: Según la media y a los parámetros establecidos en la Clasificación de Schneider en la raíz distal y raíz mesial presentan alto índice de curvatura severa, Tipo de curvatura vista raíz distal y raíz mesial dan alto índice de tipo recto, Tipo de curvatura vista vestibular raíz distal 
resultan en alto índice de tipo mesial, Tipo de curvatura vista vestibular raíz mesial se nota un índice alto de tipo distal, Curvatura raíz distal y raíz mesial según su tercio expresan resultados de alto índice de curvatura en tercio medio.

\section{Ilustración 2.- Moda}

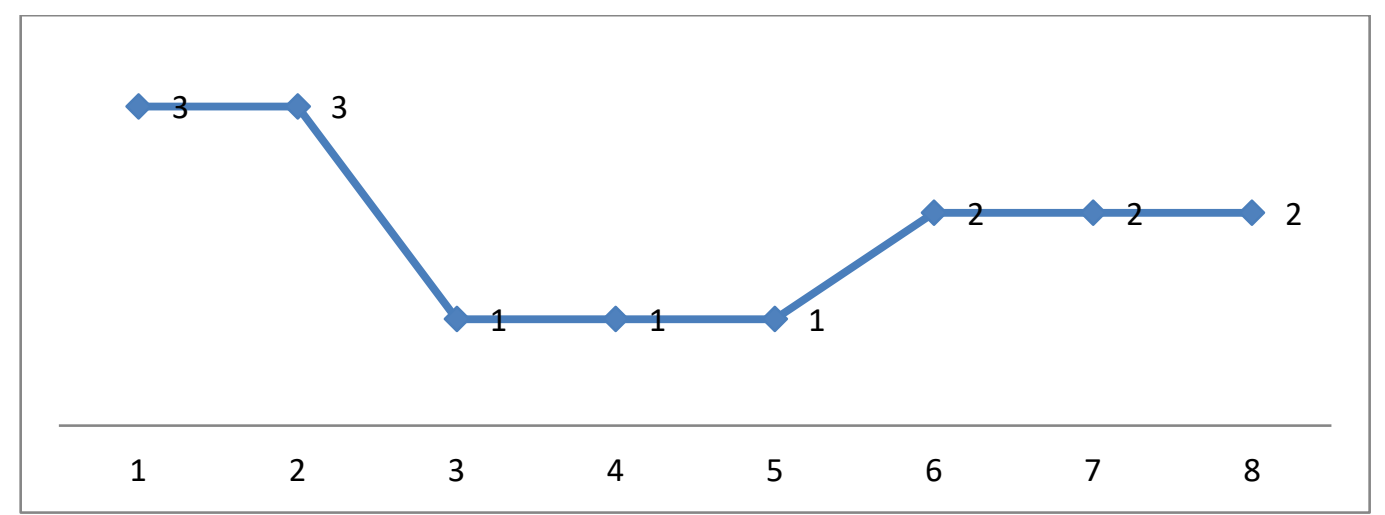

\section{Elaborado por: Roddy Chavez}

Fuente: IBM SPSS versión 22.

Descripción: La moda en referencia a cada uno de los objetivos analizados corresponde a: clasificación de Schneider en la raíz distal y mesial con un 3, Tipo de curvatura vista raíz distal, mesial es 1, Tipo de curvatura vista vestibular raíz distal 1, Tipo de curvatura vista vestibular raíz mesial 2, Curvatura raíz distal mesial según su tercio es de 2.

Análisis: En referencia a la moda y a parámetros establecidos en la Clasificación de Schneider en la raíz distal y raíz mesial se nota frecuencia alta de curvatura severa, Tipo de curvatura vista raíz distal y raíz mesial presentan frecuencia alta tipo recto, Tipo de curvatura vista vestibular raíz distal expresan resultados de frecuencia alta tipo mesial, Tipo de curvatura vista

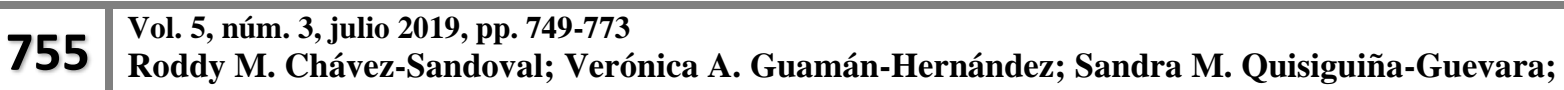

Natalia A. Gavilanes-Bayas; Cristian R. Sigcho-Romero
} 
vestibular raíz mesial expresan frecuencia alta tipo distal, Curvatura raíz distal y mesial según su tercio dan frecuencia alta de curvatura en tercio medio.

\section{Ilustración 3.- Desviación Estándar}

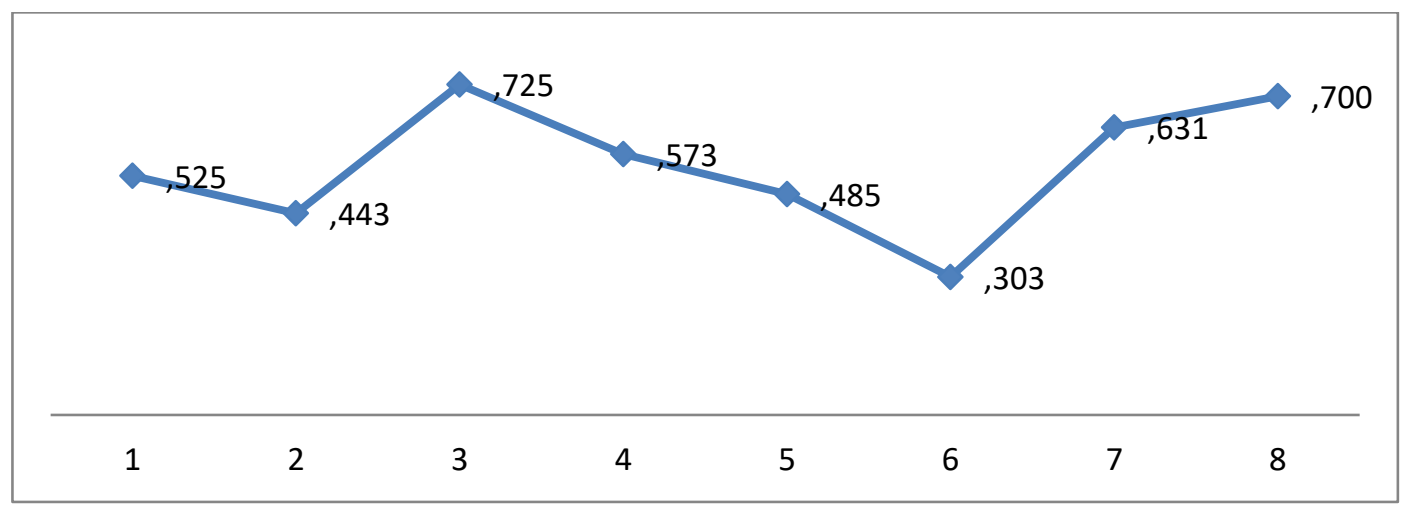

Elaborado por: Roddy Chavez

Fuente: IBM SPSS versión 22.

Descripción: Desviación estándar resultado obtenido en referencia a cada uno de los objetivos analizados corresponde a: clasificación de Schneider en la raíz distal con un 0.525 , clasificación de Schneider en la raíz mesial 0.443, Tipo de curvatura vista raíz distal 0.725, Tipo de curvatura vista raíz mesial 0.573 , Tipo de curvatura vista vestibular raíz distal 0.485 , Tipo de curvatura vista vestibular raíz mesial 0.303, Curvatura raíz distal según su tercio 0.631, Curvatura raíz mesial según su tercio 0.700 .

Análisis: Desviación estándar medida expresa dispersión de los datos con respecto a la media, con una referencia de los datos obtenidos en el que se presenta en todos los casos una desviación estándar menor lo que significa que la población está menos dispersa alrededor de la media de cada caso. 
Tabla 2.- Frecuencia Clasificación de Schneider raíz distal

\begin{tabular}{|l|l|l|l|l|l|}
\hline \multicolumn{2}{|c|}{} & Frecuencia & Porcentaje & $\begin{array}{l}\text { Porcentaje } \\
\text { válido }\end{array}$ & $\begin{array}{l}\text { Porcentaje } \\
\text { acumulado }\end{array}$ \\
\hline \multirow{4}{*}{ Válido } & Leve & 1 & 2,0 & 2,0 & 2,0 \\
\cline { 2 - 6 } & Moderada & 16 & 32,0 & 32,0 & 32,0 \\
\cline { 2 - 6 } & Severa & 33 & 66,0 & 66,0 & 100,0 \\
\hline Total & 50 & 100,0 & 100,0 & \\
\hline
\end{tabular}

Elaborado por: Roddy Chavez

Fuente: IBM SPSS versión 22.

\section{Ilustración 4.- Curvatura radicular raíz distal}

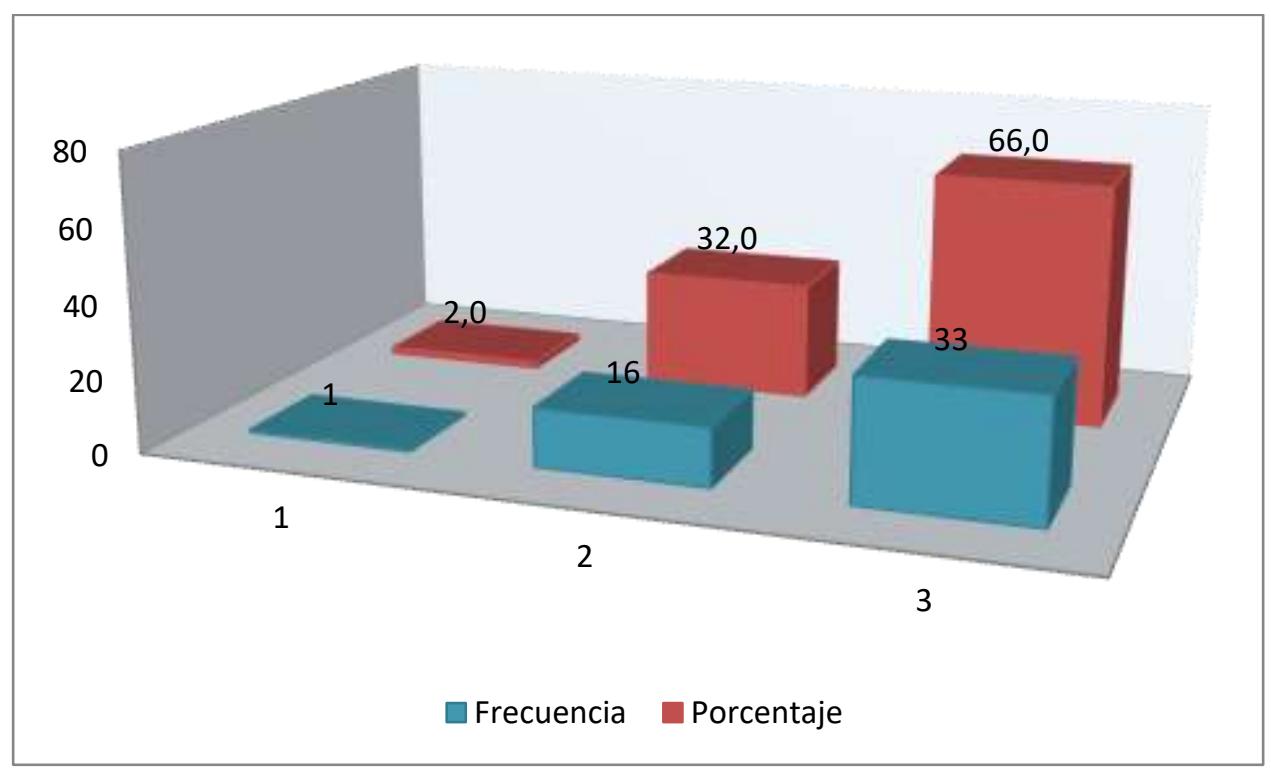

Elaborado por: Roddy Chavez

Fuente: IBM SPSS versión 22. 
Descripción: La clasificación de la curvatura radicular que se presentan en molares inferiores en radiografías peri-apicales en referencia a la raíz distal muestra una frecuencia de 1 correspondiente al 2\% de la clasificación leve, seguido de una frecuencia de 16 correspondiente al $32 \%$ de la clasificación moderada y una frecuencia de 33 correspondiente al $66 \%$ de la clasificación severa.

Análisis: Se presenta un alto porcentaje en las radiografías peri- apicales de molares inferiores en referencia a la raíz distal de un $66 \%$ de curvatura severa con una diferencia significativa sobre la curvatura leve que se presentó en un $2 \%$.

Tabla 3.- Frecuencia clasificación de Schneider raíz mesial

\begin{tabular}{|l|l|l|l|l|l|}
\hline \multicolumn{2}{|c|}{} & Frecuencia & Porcentaje & $\begin{array}{l}\text { Porcentaje } \\
\text { válido }\end{array}$ & $\begin{array}{l}\text { Porcentaje } \\
\text { acumulado }\end{array}$ \\
\hline \multirow{3}{*}{ Válido } & Moderada & 13 & 26,0 & 26,0 & 26,0 \\
\cline { 2 - 6 } & Severa & 37 & 74,0 & 74,0 & 100,0 \\
\cline { 2 - 6 } & Total & 50 & 100,0 & 100,0 & \\
\hline
\end{tabular}

Elaborado por: Roddy Chavez

Fuente: IBM SPSS versión 22. 


\section{Ilustración 5.- Curvatura radicular raíz mesial}

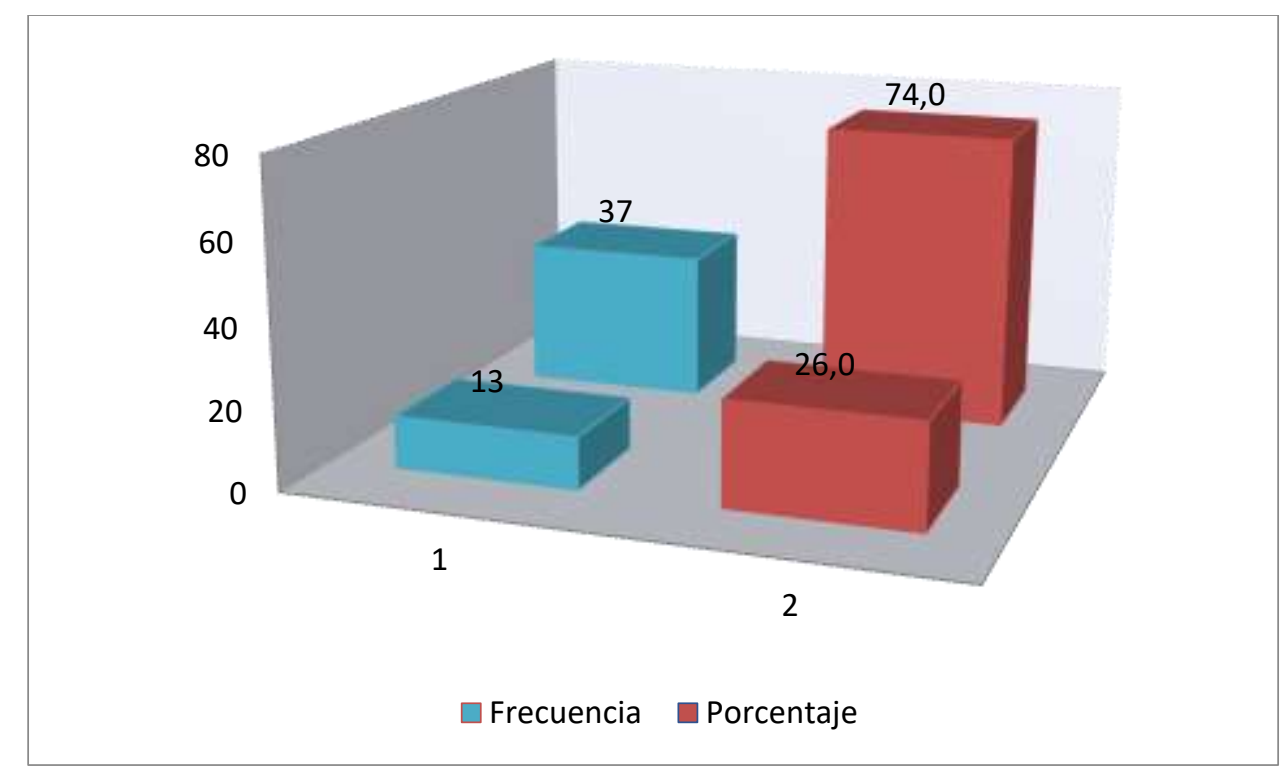

\section{Elaborado por: Roddy Chavez}

Fuente: IBM SPSS versión 22.

Descripción: La clasificación de la curvatura radicular que presentan molares inferiores en radiografías peri-apicales en referencia a la raíz mesial muestra una frecuencia de 13 correspondiente al $26 \%$ de la clasificación moderada, seguido de una frecuencia de 37 correspondiente al $74 \%$ de la clasificación severa.

Análisis: Se presenta un alto porcentaje en las radiografías periapicales de molares inferiores en referencia a la raíz mesial de un $74 \%$ de curvatura severa con una diferencia significativa sobre la curvatura moderada que se presentó en un $26 \%$ y con referencia a la curvatura leve no se presentó. 
Tabla 4.- Frecuencia tipo de curvatura vista distal

\begin{tabular}{|l|l|l|l|l|l|}
\hline \multicolumn{2}{|l|}{} & Frecuencia & Porcentaje & $\begin{array}{l}\text { Porcentaje } \\
\text { válido }\end{array}$ & $\begin{array}{l}\text { Porcentaje } \\
\text { acumulado }\end{array}$ \\
\hline Válido & Recto & 38 & 76,0 & 76,0 & 76,0 \\
\cline { 2 - 6 } & Vestibular & 5 & 10,0 & 10,0 & 86,0 \\
\cline { 2 - 6 } & Lingual & 7 & 14,0 & 14,0 & 100,0 \\
\cline { 2 - 6 } & Total & 50 & 100,0 & 100,0 & \\
\hline
\end{tabular}

Elaborado por: Roddy Chavez

Fuente: IBM SPSS versión 22.

\section{Ilustración 6.- Tipo de curvatura raíz distal}

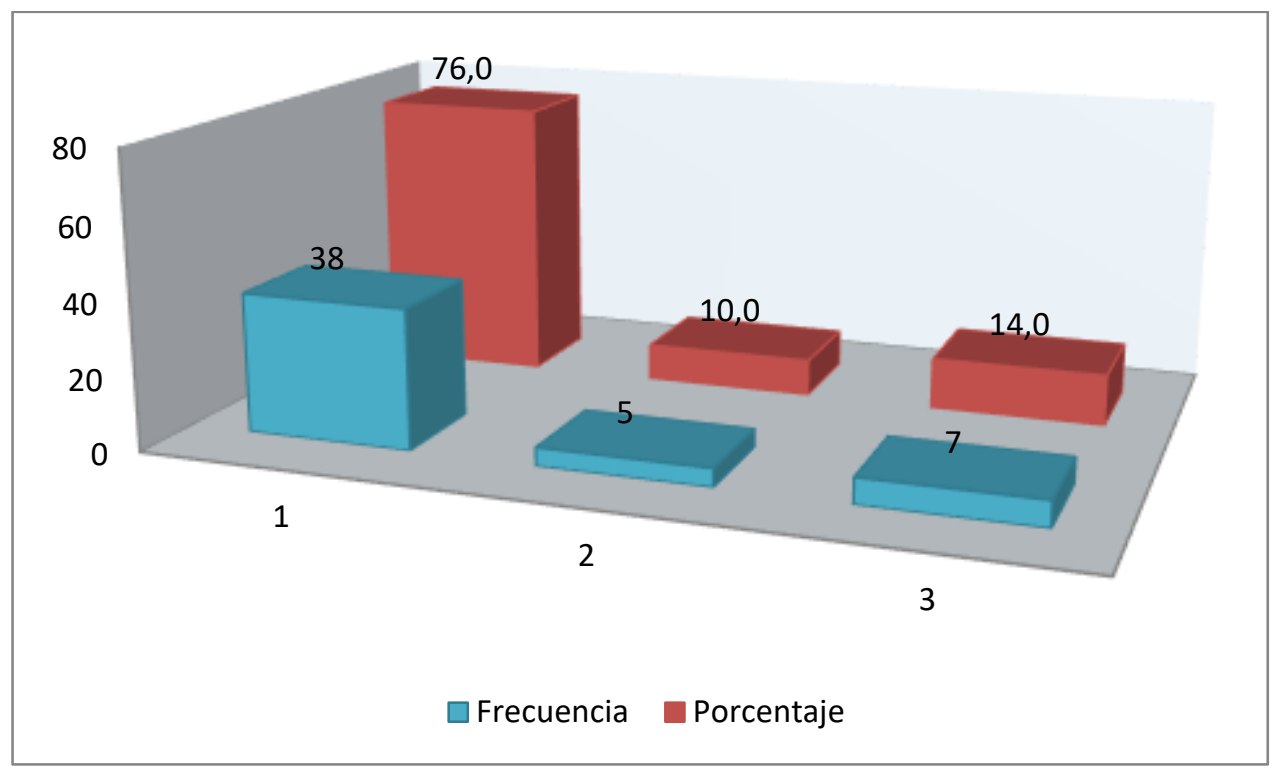

Elaborado por: Roddy Chavez

Fuente: IBM SPSS versión 23. 
Descripción: El tipo de curvatura que presentan molares inferiores en la raíz distal en radiografías peri-apicales de vista distal muestra una frecuencia de 38 correspondiente al $76 \%$ de tipo recto, seguido de una frecuencia de 5 correspondiente al $10 \%$ de tipo vestibular y una frecuencia de 7 correspondiente al $14 \%$ de tipo lingual.

Análisis: Se presenta un alto porcentaje en las radiografías peri- apicales de vista distal de molares inferiores en referencia a la raíz distal de un $76 \%$ de tipo recto con una diferencia significativa sobre el tipo vestibular y lingual que se presentan un $10 \%$ y $14 \%$ con referencia al tipo recto

Tabla 5.- Frecuencia tipo de curvatura vista mesial

\begin{tabular}{|l|l|l|l|l|l|}
\hline \multicolumn{2}{|l|}{} & Frecuencia & Porcentaje & $\begin{array}{l}\text { Porcentaje } \\
\text { válido }\end{array}$ & $\begin{array}{l}\text { Porcentaje } \\
\text { acumulado }\end{array}$ \\
\hline \multirow{4}{*}{ Válido } & Recto & 39 & 78,0 & 78,0 & 78,0 \\
\cline { 2 - 6 } & Vestibular & 8 & 16,0 & 16,0 & 94,0 \\
\cline { 2 - 6 } & Lingual & 3 & 6,0 & 6,0 & 100,0 \\
\cline { 2 - 6 } & Total & 50 & 100,0 & 100,0 & \\
\hline
\end{tabular}

Elaborado por: Roddy Chavez

Fuente: IBM SPSS versión 22. 


\section{Ilustración 7.- Tipo de curvatura raíz mesial}

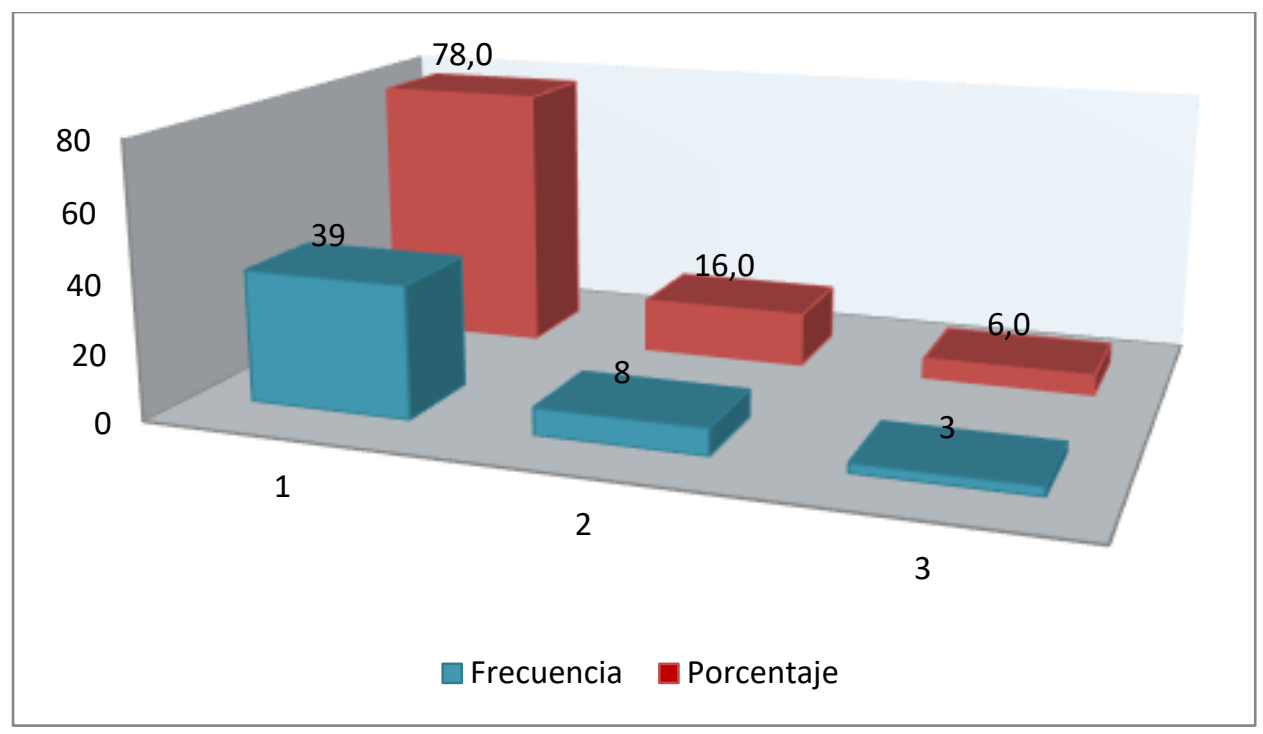

\section{Elaborado por: Roddy Chavez}

Fuente: IBM SPSS versión 23.

Descripción: El tipo de curvatura que presentan molares inferiores en la raíz mesial en radiografías peri-apicales de vista mesial muestra una frecuencia de 39 correspondiente al $78 \%$ de tipo recto, seguido de una frecuencia de 8 correspondiente al $16 \%$ de tipo vestibular y una frecuencia de 3 correspondiente al $6 \%$ de tipo lingual.

Análisis: Se presenta un alto porcentaje en las radiografías peri- apicales de vista mesial de molares inferiores en referencia a la raíz mesial de un $78 \%$ de tipo recto con una diferencia significativa sobre el tipo vestibular y lingual que se presentan un $16 \%$ y $6 \%$ con referencia al tipo recto. 
Tabla 8.- Frecuencia tipo curvatura vista vestibular raíz distal

\begin{tabular}{|l|l|l|l|l|l|}
\hline \multicolumn{2}{|c|}{} & Frecuencia & Porcentaje & $\begin{array}{l}\text { Porcentaje } \\
\text { válido }\end{array}$ & $\begin{array}{l}\text { Porcentaje } \\
\text { acumulado }\end{array}$ \\
\hline Válido & Mesial & 32 & 64,0 & 64,0 & 64,0 \\
\cline { 2 - 6 } & Distal & 18 & 36,0 & 36,0 & 100,0 \\
\cline { 2 - 6 } & Total & 50 & 100,0 & 100,0 & \\
\hline
\end{tabular}

Elaborado por: Roddy Chavez

Fuente: IBM SPSS versión 22.

\section{Ilustración 9.- Tipo de curvatura raíz distal}

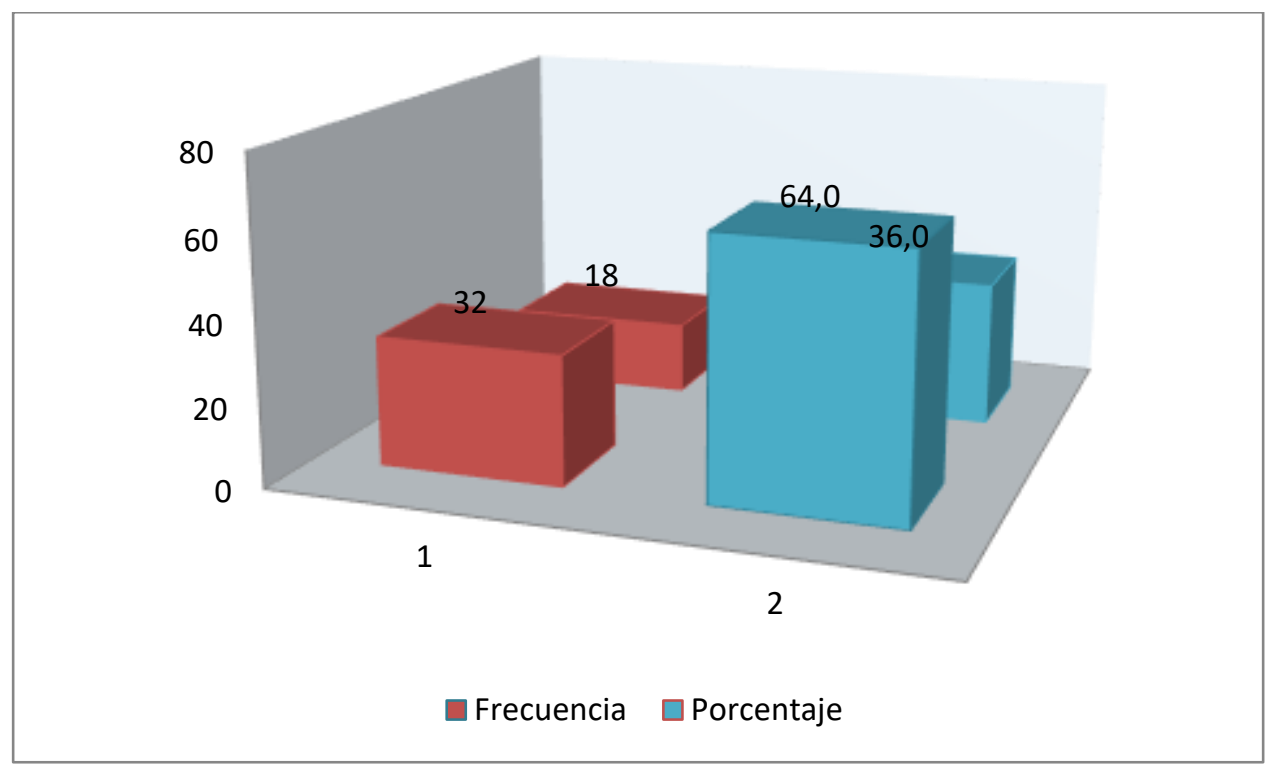

Elaborado por: Roddy Chavez

Fuente: IBM SPSS versión 22. 
Descripción: El tipo de curvatura que presentan molares inferiores en la raíz distal en radiografías peri-apicales de vista vestibular muestra una frecuencia de 32 correspondiente al $64 \%$ de tipo mesial, seguido de una frecuencia de 18 correspondiente al $36 \%$ de tipo distal.

Análisis: Se presenta una diferencia media de porcentaje en las radiografías peri- apicales de vista vestibular de molares inferiores en referencia a la raíz distal de un $64 \%$ de tipo mesial sobre el tipo distal que se presentan un $36 \%$.

Tabla 6.- Frecuencia Tipo de curvatura vista vestibular raíz mesial

\begin{tabular}{|l|l|l|l|l|l|}
\hline \multicolumn{2}{|c|}{} & Frecuencia & Porcentaje & $\begin{array}{l}\text { Porcentaje } \\
\text { válido }\end{array}$ & $\begin{array}{l}\text { Porcentaje } \\
\text { acumulado }\end{array}$ \\
\hline Válido & Mesial & 5 & 10,0 & 10,0 & 10,0 \\
\cline { 2 - 6 } & Distal & 45 & 90,0 & 90,0 & 100,0 \\
\cline { 2 - 6 } & Total & 50 & 100,0 & 100,0 & \\
\hline
\end{tabular}

Elaborado por: Roddy Chavez

Fuente: IBM SPSS versión 22. 


\section{Ilustración 10.- Tipo de Curvatura Raíz Mesial}

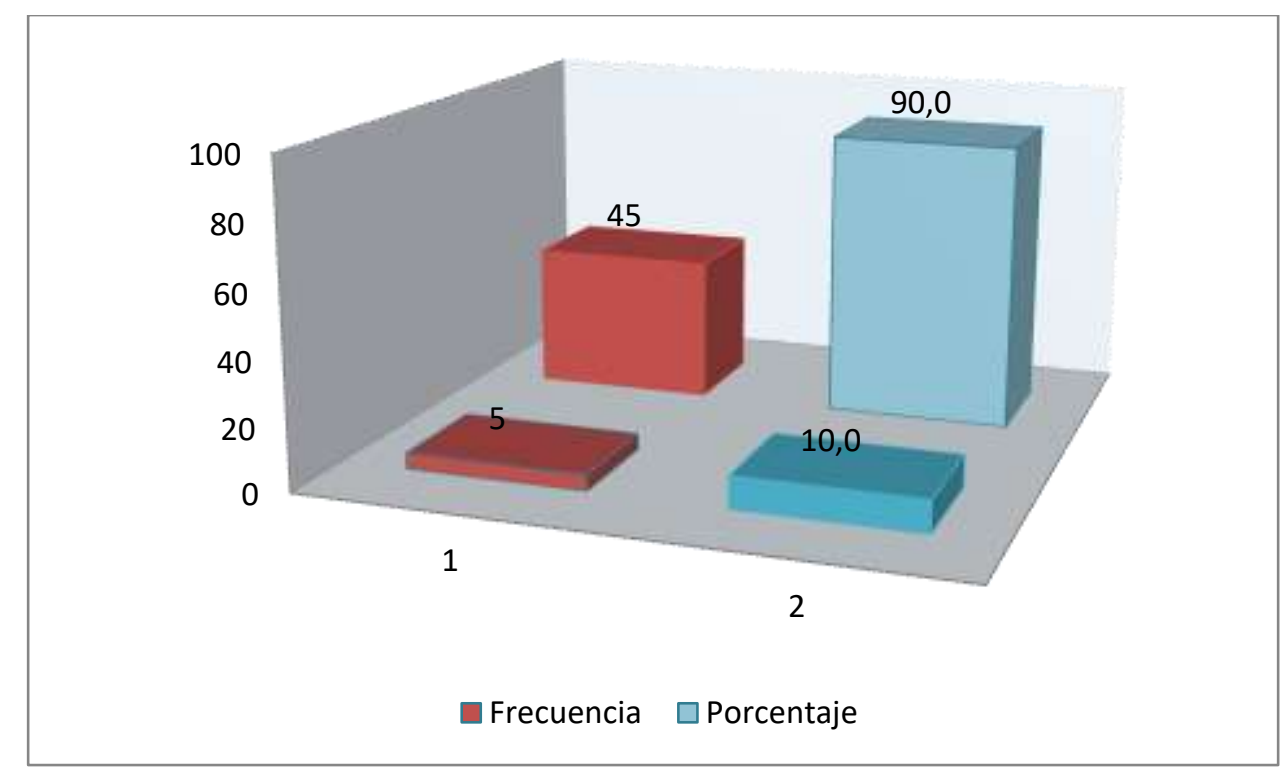

\section{Elaborado por: Roddy Chavez}

Fuente: IBM SPSS versión 22.

Descripción: El tipo de curvatura que presentan molares inferiores en la raíz mesial en radiografías peri-apicales de vista vestibular muestra una frecuencia de 5 correspondiente al $10 \%$ de tipo mesial, seguido de una frecuencia de 45 correspondiente al 90\% de tipo distal.

Análisis: Se presenta una diferencia alta de porcentaje en las radiografías peri- apicales de vista vestibular de molares inferiores en referencia a la raíz mesial de un $90 \%$ de tipo distal sobre el tipo distal que se presentan un $10 \%$.

Tabla 11.- Frecuencia de curvatura raíz distal según su tercio

\begin{tabular}{|l|l|l|l|l|}
\hline & Frecuencia & Porcentaje & $\begin{array}{l}\text { Porcentaje } \\
\text { válido }\end{array}$ & $\begin{array}{l}\text { Porcentaje } \\
\text { acumulado }\end{array}$ \\
\hline
\end{tabular}


Análisis de la curvatura radicular para definir tratamiento endodontico de molares inferiores. Universidad Nacional de Chimborazo, 2018

\begin{tabular}{|l|l|l|l|l|l|}
\hline Válido & Coronal & 4 & 8,0 & 8,0 & 8,0 \\
\cline { 2 - 6 } & Medio & 24 & 48,0 & 48,0 & 56,0 \\
\cline { 2 - 6 } & Apical & 22 & 44,0 & 44,0 & 100,0 \\
\cline { 2 - 6 } & Total & 50 & 100,0 & 100,0 & \\
\hline
\end{tabular}

Elaborado por: Roddy Chavez

Fuente: IBM SPSS versión 22.

\section{Ilustración 12.-Curvatura raíz distal según su tercio}

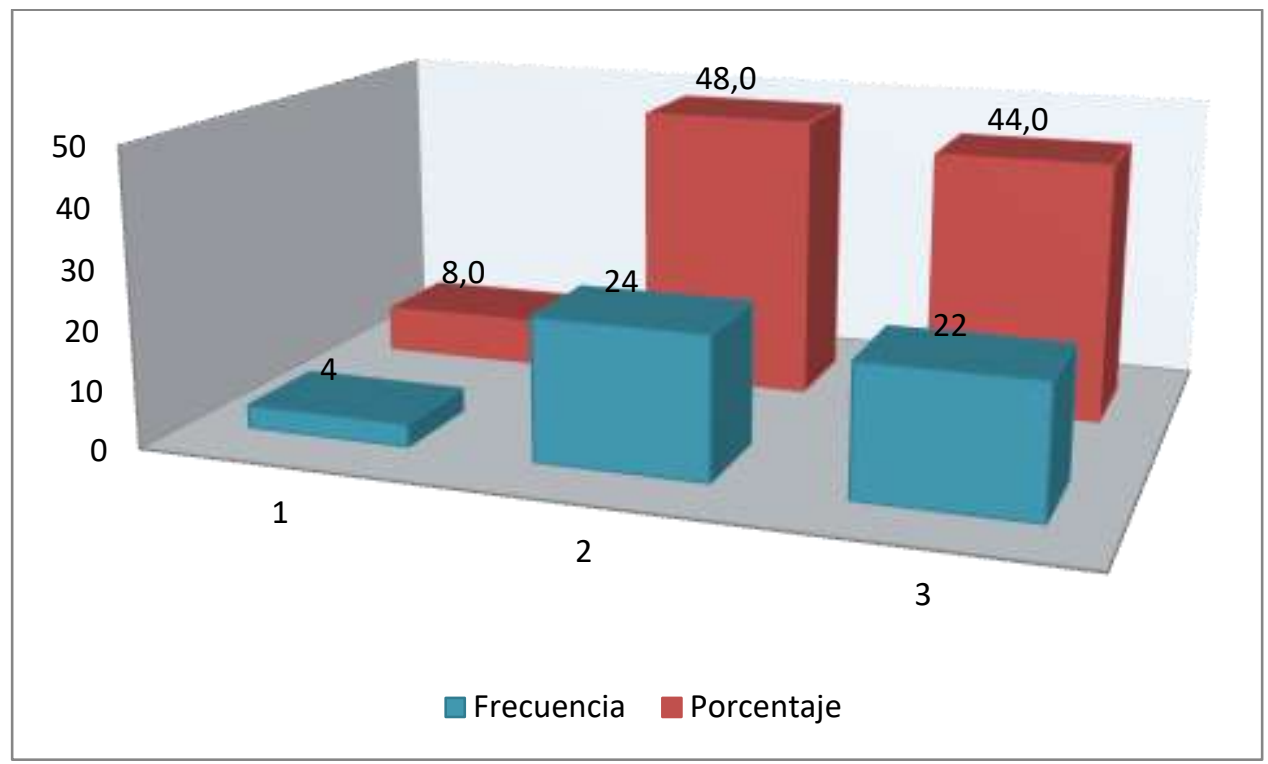

Elaborado por: Roddy Chavez

Fuente: IBM SPSS versión 22.

Descripción: La curvatura de la raíz distal según su tercio en molares inferiores en radiografías peri-apicales muestra una frecuencia de 4 correspondiente al $8 \%$ con curvatura en tercio 
coronal, seguido de una frecuencia de 24 correspondiente al $48 \%$ de curvatura en tercio medio y una frecuencia de 22 correspondiente al $44 \%$ de curvatura en tercio apical.

Análisis: Se presenta un alto porcentaje en las radiografías peri- apicales de molares inferiores en referencia a la raíz distal de un $48 \%$ de curvatura en tercio medio con una diferencia significativa sobre la curvatura en el tercio coronal que se presentan un $8 \%$ y porcentaje medio sobre la curvatura en el tercio apical con un $44 \%$.

Tabla 8.- Frecuencia de curvatura raíz mesial según su tercio

\begin{tabular}{|l|l|l|l|l|l|}
\hline \multicolumn{2}{|c|}{} & Frecuencia & Porcentaje & $\begin{array}{l}\text { Porcentaje } \\
\text { válido }\end{array}$ & $\begin{array}{l}\text { Porcentaje } \\
\text { acumulado }\end{array}$ \\
\hline \multirow{4}{*}{ Válido } & Coronal & 16 & 32,0 & 32,0 & 32,0 \\
\cline { 2 - 6 } & Medio & 25 & 50,0 & 50,0 & 82,0 \\
\cline { 2 - 6 } & Apical & 9 & 18,0 & 18,0 & 100,0 \\
\cline { 2 - 6 } & Total & 50 & 100,0 & 100,0 & \\
\hline
\end{tabular}

Elaborado por: Roddy Chavez

Fuente: IBM SPSS versión 22. 


\section{Ilustración 13.- Curvatura raíz mesial según su tercio}

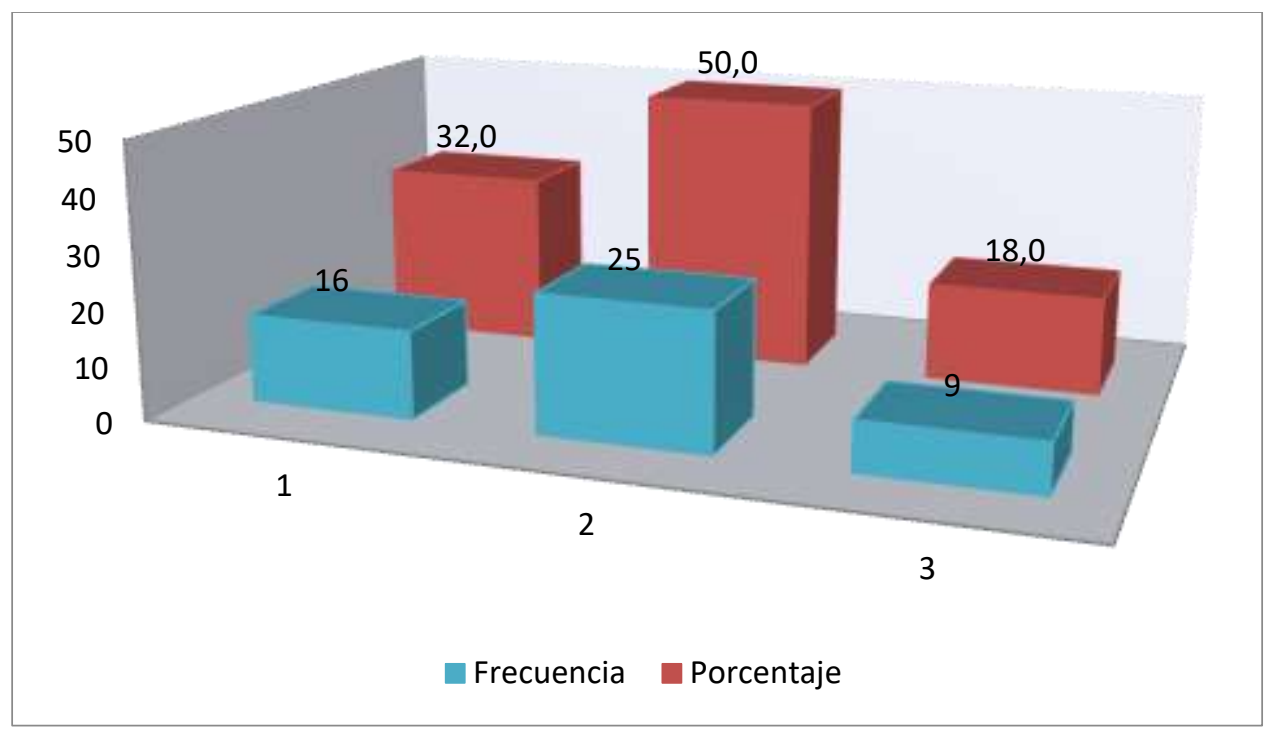

\section{Elaborado por: Roddy Chavez}

Fuente: IBM SPSS versión 22.

Descripción: La curvatura de la raíz mesial según su tercio en molares inferiores en radiografías peri-apicales muestra una frecuencia de 16 correspondiente al $32 \%$ con curvatura en tercio coronal, seguido de una frecuencia de 25 correspondiente al 50\% de curvatura en tercio medio y una frecuencia de 9 correspondiente al $18 \%$ de curvatura en tercio apical.

Análisis: Se presenta un alto porcentaje en las radiografías peri- apicales de molares inferiores en referencia a la raíz mesial de un 50\% de curvatura en tercio medio con una diferencia significativa sobre la curvatura en el tercio apical que se presentan un $18 \%$ y porcentaje medio sobre la curvatura en el tercio coronal con un $32 \%$. 


\section{Tabla 9.-Prueba de Wilcoxon}

\begin{tabular}{|l|l|l|l|l|}
\hline \multicolumn{1}{|l|}{ Estadísticos de prueba } \\
\hline & $\begin{array}{l}\text { Clasificación de } \\
\text { Schneider raíz mesial } \\
-\quad \text { Clasificación de } \\
\text { Schneider raíz distal }\end{array}$ & $\begin{array}{l}\text { Vista } \\
\text { mesial } \\
\text { Vista distal }\end{array}$ & $\begin{array}{l}\text { Vista vestibular } \\
\text { raíz mesial - Vista } \\
\text { vestibular raíz } \\
\text { distal }\end{array}$ & $\begin{array}{l}\text { Tercio raíz } \\
\text { mesial - Tercio } \\
\text { raíz distal }\end{array}$ \\
\hline $\begin{array}{l}\text { Sig. } \\
\text { asintótica } \\
\text { (bilateral) }\end{array}$ & 0,34 & 0,19 & 0,000 & 0,004 \\
\hline
\end{tabular}

Elaborado por: Roddy Chavez

Fuente: IBM SPSS versión 22.

Descripción: La clasificación de Schneider raíz mesial en comparación con la clasificación de Schneider raíz distal recibió un $\mathrm{P}$ valor de 0.34 , la vista mesial sobre la vista distal de tipo de curvatura presenta un $\mathrm{P}$ valor de 0.19 , la vista vestibular de la raíz mesial sobre la vista vestibular raíz distal presenta un $\mathrm{P}$ valor de 0.000 y el tercio de raíz mesial en el que se presenta la curvatura sobre el tercio de raíz distal en el que se presenta la curvatura es de un P valor 0.004

Análisis: La clasificación de Schneider raíz mesial en comparación con la clasificación de Schneider raíz distal presentan un valor no significativo, la vista mesial sobre la vista distal de tipo de curvatura presentan un valor no significativo, la vista vestibular de la raíz mesial sobre la vista vestibular raíz distal presenta un valor significativo y el tercio de raíz mesial en el que se presenta la curvatura sobre el tercio de raíz distal en el que se presenta la curvatura es de un valor muy significativo. 


\section{Discusión.}

En el presente estudio se observa que la frecuencia de la curvatura de la raíz distal se representó en 1 correspondiente al 2\% de clasificación leve, el 16 correspondiente al 32\% de clasificación moderada, el 33 correspondiente al 66\% de clasificación severa, en consecuencia a la raíz mesial se obtuvo una frecuencia de 13 correspondiente al $26 \%$ de clasificación moderada, el 37 correspondiente al 74\% de la clasificación severa.

Estos datos se presentan de forma parecida al estudio de morfometría de Premolares mandibular en radiografías Panorámicas Digitales; Análisis de Curvaturas Radiculares, realizadas en radiografías panorámicas, el 71,08\% de los dientes analizados presenta algún grado de curvatura. Con una semejanza al estudio de Llena et al. (2014), quien utilizando imágenes 3D, presentaron el $65 \%$ de premolares mandibulares analizados presentaron grado de curva en su raíz. (5)

Se observar en el tipo de curvatura raíz distal tiene una frecuencia tipo recto de 38 que corresponde al 76\%, tipo vestibular con una frecuencia de 5 correspondiente al $10 \%$ y de 7 correspondiente al 14\% tipo lingual, el tipo de curvatura raíz mesial muestra una frecuencia tipo recto de 39 correspondiente al 78\%, 8 tipo vestibular correspondiente al 16\% y 3 correspondiente al $6 \%$ de tipo lingual.

El tipo de curvatura en la raíz distal desde una vista vestibular muestra una frecuencia de 32 correspondiente al $64 \%$ de tipo mesial, frecuencia de 18 correspondiente al 36\% de tipo distal, la curvatura que presentan en la raíz mesial vista vestibular muestra una frecuencia de 5 correspondiente al 10\% de tipo mesial, frecuencia de 45 correspondiente al $90 \%$ de tipo distal.

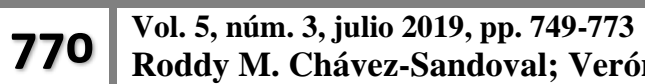
Roddy M. Chávez-Sandoval; Verónica A. Guamán-Hernández; Sandra M. Quisiguiña-Guevara; 
En la investigación Prevalencia de curvaturas radiculares en dientes maxilares anteriores tratados endodonticamente Clínica UCSG año 2013 - 2016 dio como resultado la dirección del tipo curvatura radicular en dientes laterales medidas radiográficamente dio como resultado el $86 \%$ rectos, el 2\% Mesial y el 12\% distal, en dientes caninos el tipo de curvatura radicular dio medidas radiográficamente del $60 \%$ rectos y el $40 \%$ distal. (6)

En este estudio también podemos ver que la curvatura de la raíz distal según su tercio presenta una frecuencia de 4 correspondiente al $8 \%$ con curvatura en tercio coronal, seguido de una frecuencia de 24 correspondiente al $48 \%$ de curvatura en tercio medio y una frecuencia de 22 correspondiente al $44 \%$ de curvatura en tercio apical, en la curvatura de la raíz mesial según su tercio muestra una frecuencia de 16 correspondiente al $32 \%$ con curvatura en tercio coronal, seguido de una frecuencia de 25 correspondiente al 50\% de curvatura en tercio medio y una frecuencia de 9 correspondiente al 18\% de curvatura en tercio apical.

El tercio radicular con curvatura en dientes laterales medidos radiográficamente arrojo resultados de $86 \%$ no tienen curvatura, mientras que el $2 \%$ tiene tercio medio y el $12 \%$ corresponde al tercio apical, El tercio radicular con curvatura en dientes caninos dio como resultado que el $60 \%$ no tienen curvatura, mientras que el $40 \%$ tiene tercio apical. (6)

Las curvaturas de los molares inferiores que se pueden presentar según Dr. Ricardo Rivas en dirección recta raíz mesial 27\% y distal 58\%, curva hacia distal raíz mesial $61 \%$ y distal 18\%, curva hacia mesial raíz mesial 0 y distal $10 \%$, curva hacia vestibular raíz mesial $4 \%$ y distal $4 \%$, curva hacia lingual raíz mesial 0 y distal 0, dilaceración marcada hacia mesial 7\% y distal 6\%. (7)

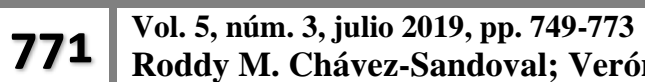
erónica A. Guamán-Hernández; Sandra M. Quisiguiña-Guevara; Natalia A. Gavilanes-Bayas; Cristian R. Sigcho-Romero
} 


\section{Conclusiones.}

- Mediante el análisis de las radiografías peri-apicales de varias vistas con la técnica de Schneider y observación se ha llegado a las siguientes conclusiones:

- Mediante la técnica de Schneider realizada en AutoCAD versión 2015 concluye que en un gran porcentaje la raíz distal y mesial del molar inferior tienen curvatura severa.

- El tipo de curvatura radicular recto es el más frecuente en la población tanto como de la vista distal y mesial de la radiografía peri-apical.

- El tipo curvatura radicular en la vista vestibular de la radiografía peri-apical presenta un alto porcentaje de la raíz mesial con curvatura hacia distal y de la raíz distal con curvatura hacia mesial.

- La curvatura según su tercio radicular expresan frecuencia alta en la raíz distal con curvatura en el tercio medio y apical y en la raíz mesial es alta con curvatura en tercio medio.

\section{Referencias bibliográficas.}

1. Tratamiento de conducto [Internet]. [citado 24 de octubre de 2018]. Disponible en: https://contenidos.bupasalud.com/salud-bienestar/vida-bupa/tratamiento-de-conducto.

2. Estudio de prevalencia de curvaturas radiculares en caninos superiores y su importancia en los tratamientos de endodoncia. - PDF [Internet]. [citado 24 de octubre de 2018]. Disponible

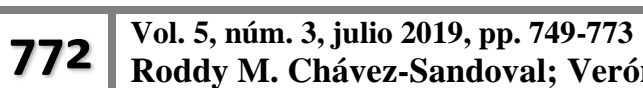
Roddy M. Chávez-Sandoval; Verónica A. Guamán-Hernández; Sandra M. Quisiguiña-Guevara; Natalia A. Gavilanes-Bayas; Cristian R. Sigcho-Romero
} 
en: $\quad$ https://docplayer.es/79927059-Estudio-de-prevalencia-de-curvaturas-radiculares-encaninos-superiores-y-su-importancia-en-los-tratamientos-de-endodoncia.html.

3. DocAnatomiaDeMolares.pdf [Internet]. [citado 24 de octubre de 2018]. Disponible en: http://www.postgradosodontologia.cl/endodoncia/images/EspecialidadEndodoncia/Seminari os/2013-2014/DocAnatomiaDeMolares.pdf

4. 5.pdf [Internet]. [citado 12 de febrero de 2019]. Disponible en: http://132.248.9.34/hevila/Odontologiaactual/2008/vol6/no61/5.pdf

5. Fuentes R, Arias A, Navarro P, Ottone N, Bucchi C. Morfometría de Premolares Mandibulares en Radiografías Panorámicas Digitales; Análisis de Curvaturas Radiculares. Int J Morphol. junio de 2015;33(2):476-82.

6. Estudio de prevalencia de curvaturas radiculares en caninos superiores y su importancia en los tratamientos de endodoncia. - PDF [Internet]. [citado 24 de octubre de 2018]. Disponible en: https://docplayer.es/79927059-Estudio-de-prevalencia-de-curvaturas-radiculares-encaninos-superiores-y-su-importancia-en-los-tratamientos-de-endodoncia.html

7. Morfología $2^{\circ}$ molar inferior [Internet]. [citado 14 de diciembre de 2018]. Disponible en: http://www.iztacala.unam.mx/rrivas/NOTAS/Notas2Morfologia/morfologia47.html

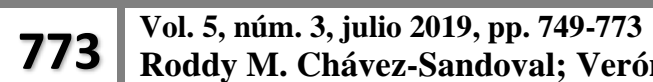
Roddy M. Chávez-Sandoval; Verónica A. Guamán-Hernández; Sandra M. Quisiguiña-Guevara; Natalia A. Gavilanes-Bayas; Cristian R. Sigcho-Romero
} 